\title{
Molecular Dynamics Simulations of the STAS Domains of Rat Prestin and Human Pendrin Reveal Conformational Motions in Conserved Flexible Regions
}

\author{
Alok K. Sharma ${ }^{a}$ Israel Zelikovic ${ }^{b} \quad$ Seth L. Alper \\ aRenal Division, Division of Molecular and Vascular Medicine, Center for Vascular Biology Research, \\ Beth Israel Deaconess Medical Center, Department of Medicine, Harvard Medical School, Boston, MA,

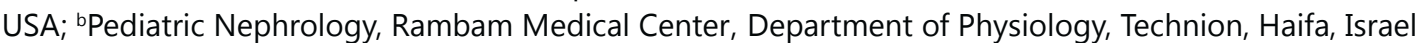

\section{Key Words}

STAS domain • Rat prestin • Human pendrin • Molecular dynamics simulation • Atomic motions - RMSD $\cdot$ RMSF • Conformational change

\begin{abstract}
Background: Molecular dynamics (MD) simulations provide valuable information on the conformational changes that accompany time-dependent motions in proteins. The reported crystal structure of rat prestin (PDB 3LLO) is remarkable for an $\alpha 1-\alpha 2$ inter-helical angle that differs substantially from those observed in bacterial STAS domains of SulP anion transporters and anti-sigma factor antagonists. However, NMR data on the rat prestin STAS domain in solution suggests dynamic features at or near the $\alpha 1-\alpha 2$ helical region (Pasqualetto et al $J M B, 2010)$. We therefore performed a 100 ns $300 \mathrm{~K}$ MD simulation study comparing the STAS domains of rat prestin and (modeled) human pendrin, to explore possible conformational flexibility in the region of the $\alpha 1$ and $\alpha 2$ helices. Methods: The conformation of the loop missing in the crystal structure of rat prestin STAS ( 11 amino acids between helix $\alpha 1$ and strand $\beta 3$ ) was built using Modeller. MD simulations were performed with GROMACSv4.6 using GROMOS96 $53 a 6$ all-atom force field. Results: A subset of secondary structured elements of the STAS domains exhibits significant conformational changes during the simulation time course. The conformationally perturbed segments include the majority of loop regions, as well as the $\alpha 1$ and $\alpha 2$ helices. A significant decrease in the $\alpha 1-\alpha 2$ inter-helical angle observed across the simulation trajectory leads to closer helical packing at their C-termini. The end-simulation conformations of the prestin and pendrin STAS domains, including their decreased $\alpha 1-\alpha 2$ inter-helical angles, resemble more closely the packing of corresponding helices in the STAS structures of bacterial SulP transporters Rv1739c and ychM, as well as those of the anti-sigma factor antagonists. Several structural segments of the modeled human pendrin STAS domain exhibit larger atomic motions and greater conformational deviations than the corresponding regions of rat prestin, predicting that the human pendrin STAS domain in solution structure
\end{abstract}




\section{Cellular Physiology and Biochemistry}

Cell Physiol Biochem 2014;33:605-620

DOI: $10.1159 / 000358638$

Published onlıne: February 27, 2014

c) 2014 S. Karger AG, Basel

www.karger.com/cpb

Sharma/Zelikovic/Alper: Molecular Dynamics Simulations of Mammalian STAS Domains

may be more dynamic than rat prestin STAS. Regions of prestin and pendrin identified by RMS fluctuation data as exhibiting larger atomic fluctuations corresponded to nominal GDP-binding regions of the aligned Rv1739c STAS domain of M. tuberculosis. Conclusions: MD simulations of mammalian STAS domains reveal substantial predicted conformational heterogeneity. These predicted conformational dynamics serve to supplement the reported crystal structure of the rat prestin STAS domain, and extend our understanding of the roles of STAS domains in SLC26 anion transporter function.

Copyright (C) 2014 S. Karger AG, Basel

\section{Introduction}

The $\underline{\text { Solute }}$ Carrier 26 (SLC26) gene superfamily is conserved throughout phylogeny [14]. SLC26 genes encode polypeptides that function as electroneutral or electrogenic anion exchangers of monovalent and/or divalent anions, and in some cases as anion channels. Structurally, SLC26 polypeptides are characterized by N-terminal cytoplasmic domains, 10-14 hydrophobic transmembrane spans traversing the lipid bilayer, and a C-terminal cytoplasmic "sulfate transporter and anti-sigma-factor-antagonist" (STAS) domain $[3,5]$. The eleven mammalian SLC26 genes (SLC26A1-11) exhibit varied tissue distribution patterns of expression [4]. Four of the human SLC26 genes are associated with the Mendelian recessive diseases of chondrodysplasia (SLC26A2), chloride diarrhea (SLC26A3), deafness with incompletely penetrant later-onset goiter (SLC26A4), and asthenozoospermia (SLC26A8) [6]. SLC26A4 is expressed in the apical membrane of cochlear and vestibular epithelial cells. SLC26A5/prestin is expressed in the basolateral membrane of cochlear outer hair cells, where it acts as a mechanotransducer to generate the non-linear capacitance response. Although genetic inactivation of prestin in the mouse causes hearing loss, deafness has not been convincingly associated with prestin mutations in humans.

STAS domains are also present in the large group of homologous SulP sulfate/anion transporters of plants, yeast, and bacteria [2]. The STAS domains of bacterial SulP anion transport proteins subjected to structural analysis include STAS domains of E. coli ychM (in spontaneous complex with malonyl-coA-liganded acyl carrier protein) [7] and of $M$. tuberculosis Rv1739c [8, 9] shown to bind guanine nucleotides [10]. Prokaryotic STAS domains differ from eukaryotic STAS domains in lacking the nominally unstructured "intervening variable sequence" (IVS) present in the latter. Indeed, the crystallized rat prestin STAS domain encompassing prestin aa 505-718 (Uniprot Q9EPH0) was a construct from which the 73 aa IVS region between helix $\alpha 1$ and strand $\beta 3$, was deleted [11]. The NMR secondary structure of rat prestin STAS reported in the same publication (BMRB entry 16910) lacked resonance assignment for helix $\alpha 1$ and part of the loop immediately preceding helix $\alpha 2$, perhaps due to resonance broadening beyond detection. The differential distribution of crosspeak intensities noted in the ${ }^{1} \mathrm{H}-{ }^{15} \mathrm{~N} 2 \mathrm{D}$ HSQC spectrum was also consistent with intrinsic dynamic features of protein conformation. Comparison of STAS domain structures reveals that packing of helix $\alpha_{1}$ with respect to helix $\alpha_{2}$ is strikingly different in rat prestin [11] than in the two bacterial SulP STAS structures [7, 9]. The $\alpha_{1}-\alpha_{2}$ inter-helical angle described by the two helical axes in rat prestin STAS is substantially greater than in the STAS domains of Rv1739c and ychM. The $\alpha_{1}-\alpha_{2}$ inter-helical angles of bacterial SulP STAS domains closely resemble those found in structures of the prototype STAS domains, the anti-sigma factor antagonists of $B$. subtilis SpoIIAA in unphosphorylated [12] and phosphorylated [13] forms or in complex with ADPor ATP-liganded anti-sigma factor SpoIIAB [14], or TM1442 from T. maritime [15].

To better understand the structural and motional differences between the STAS domains of rat prestin and the bacterial SulP proteins, we conducted $100 \mathrm{~ns}$ molecular dynamics (MD) simulation studies of the STAS domains of rat prestin (SLC26A5) and human pendrin (SLC26A4). Proteins exhibit dynamic motions across a wide range of time scales and transitions among various conformations which are considered an important part of protein function. MD simulations provide access to understanding time-dependent dynamic 


\section{Cellular Physiology and Biochemistry}

Cell Physiol Biochem 2014;33:605-620

\begin{tabular}{l|l}
\hline DOI: $10.1159 / 000358638$ & (c) 2014 S. Karger AG, Basel
\end{tabular}

www.karger.com/cpb

behavior of localized residues and rigid body sub-domains of proteins [16]. We hypothesized that such simulation studies might address the possible contributions to determination of the STAS domain $\alpha_{1}-\alpha_{2}$ inter-helical angles by the 11 additional aa residues not visualized in the rat prestin structures [11]. We also hypothesized a possible relationship between the most mobile residues of rat prestin STAS domain with the guanine nucleotide-interacting residues of M. tuberculosis Rv1739c STAS domain [10] which tended to exhibit increased mobility [9].

The substrate for our molecular dynamics simulations was the crystal structure of rat prestin, modified by modeling in the 11 loop residues missing from the electron density map [11]. This modified prestin structure was the template on which human pendrin was then modeled.

\section{Materials and Methods}

\section{Molecular Modeling}

The crystal structure of rat prestin STAS domain (PDB ID 3LLO) was reported for a 143 aa construct encompassing prestin aa 505-718 that excluded aa 564-636, the 73 aa IVS region between helix $\alpha_{1}$ and strand $\beta_{3}$ [11]. Missing from the electron density map of this prestin STAS construct crystal (and thus missing from the solved structure) were eleven construct residues: 556-LeuLysArgLysThrGlyValAsn-563, a GlySer pair introduced by the processes of IVS deletion and ORF religation, and Glu-637.

These 11 missing residues between helix $\alpha_{1}$ and strand $\beta_{3}$ were modeled to obtain a more complete structure of rat prestin STAS. The missing residues exhibited low values of $\left\{{ }^{1} \mathrm{H}\right\}^{-15} \mathrm{~N}$ heteronuclear NOEs and predicted order parameter $\left(S^{2}\right)$, consistent with a loop conformation[11]. The Chemical Shift Index (CSI) [17] calculated using reported NMR chemical shifts of (BMRB 16910) validated the prediction of a loop conformation for these 11 residues, consistent with elevated flexibility apparent in both solution and crystal [11]. Modeling was performed with Modeller v9.9 [18]. The modeling calculations and later dynamics simulations included $\beta$-octyl-glucoside ( $\beta$-OG), which co-crystallized with prestin [11], to maintain a modeling environment consistent with available structural information and crystallization conditions. DOPE-based loop modeling was optimized to determine 100 structural models by satisfying spatial restraints of bond lengths, bond angles, and improper dihedral angles. A subset of loop models was subsequently refined through selection based on global low DOPE potentials, energy minimization to avoid steric clashes, and 500 ps MD simulation in Gromacsv4.6. The five models that best preserved secondary and tertiary structures and exhibited high loop stability, narrow oscillation window of RMS deviation, and good stereochemical quality were subjected to a second round of energy minimization in Gromacs [19] and selected for further study.

An amino acid sequence alignment of rat prestin and human pendrin (Uniprot ID 043511) generated with ClustalW2 (http://www.ebi.ac.uk/Tools/msa/clustalw2/) and as reported [11, 20] yielded a 143 aa human pendrin STAS domain encompassing aa 515-734 (Uniprot numbering) that excluded 79 aa long IVS region (from aa 574-652) between helix $\alpha_{1}$ and strand $\beta_{3}$ (structure labeling of PDB 3LLO). The STAS domain of human pendrin was modeled using as template the best of the five highest quality loopmodeled structures of rat prestin STAS domain. The five best pendrin STAS models were selected from 100 structural models generated using the same selection criteria as described above for rat prestin STAS. All structural models were energy minimized using the steepest-descent method with 1,000 steps of step size $0.01 \mathrm{~nm}$. Stereochemical quality of these models was judged based on low $z$-score, best $(\phi, \psi)$ score in the Ramachandran map, and fewest atomic clashes as assessed by PROCHECK [21], PROSA [22], VERIFY3D [23], and PSVS server (http://psvs-1_5-dev.nesg.org/). Crystal packing in PDB 3LLO was performed using programs Coot v0.6.2 [24] and CryCo5_1 [25].

\section{Molecular dynamics}

MD simulations were performed with GROMACSv4.6 or v4.6.4 [19] using GROMOS96 53a6 all-atom force field [26] on either an 8-processor Linux PC running Intel Xeon or on a 12-processor linux PC on Orchestra clusters (Harvard Medical School Research computing). Simulations were performed for each of five best structural models selected for the STAS domains of rat prestin and human pendrin. Each 


\section{Cellular Physiology and Biochemistry}

Cell Physiol Biochem 2014;33:605-620

\begin{tabular}{l|l}
\hline DOI: $10.1159 / 000358638$ & (C) 2014 S. Karger AG, Basel
\end{tabular}

www.karger.com/cpb

Sharma/Zelikovic/Alper: Molecular Dynamics Simulations of Mammalian STAS Domains

loop-modeled crystal structure of rat prestin STAS and each modeled structure of human pendrin STAS was subjected to $100 \mathrm{~ns}$ dynamics simulations, requiring 172-214 hrs of run time. $\beta$-OG topology was parameterized to achieve force field compatibility, as described [27]. Protein structures were passed to the "WHAT If" server (http://swift.cmbi.ru.nl/servers/html/index.html) to complete the missing atom(s) before starting the simulations. Protein molecules were solvated explicitly with SPC water molecules and were inserted in a periodic, dodecahedral box of minimum distance $1.2 \mathrm{~nm}$ from center.

Standard ionization states were used for Lys, Arg, Glu, and Asp residues. The non-protonated state of Gln residues was used. Protonation states of individual His residues were chosen based on the hydrogen bonding patterns (on N $\delta 1, \mathrm{~N} \varepsilon 1$, or both) identified in the structural models. Programs Coot v0.6.2 [24], H++ v3.1 [28], MOE [29], and SCWRL [30] were used for structural inspection. The termini of the protein were kept uncharged. Appropriate numbers of water molecules were replaced with $\mathrm{Na}^{+}$and $\mathrm{Cl}^{-}$ions to achieve system electroneutrality at $150 \mathrm{mM} \mathrm{NaCl}$. The net charge of -8 units for rat prestin STAS incurred replacement of 66 water molecules with $37 \mathrm{Na}^{+}$and $29 \mathrm{Cl}^{-}$ions. 9224 water molecules were required to fill the $5.2 \times 3.8 \times 3.5 \mathrm{~nm}$ simulation box. The net charge of the human pendrin STAS domain was zero. Its 4.5 x 3.8 x $4.5 \mathrm{~nm}$ simulation box required 8982 water molecules, $27 \mathrm{Na}^{+}$ions, and $27 \mathrm{Cl}^{-}$ions. Structures were energy-minimized using a steepest-descent algorithm. Convergence was achieved in 587 and 509 steps (step size $0.01 \mathrm{~nm}$ ) for rat prestin and human pendrin, respectively. A $300 \mathrm{~K}$ reference temperature was set throughout the simulation using a Berendsen thermostat with a time constant of $0.1 \mathrm{ps}$. Bond-lengths were constrained by the LINCS algorithm [31]. Before production runs, the system was equilibrated in two steps, with 250 ps NVT simulations in the absence of pressure-coupling followed by 250 ps NPT simulations in the presence of Perrinello-Rahman pressure-coupling. Position restraints were applied to the protein molecule during equilibration. A time step of 2 fs was selected. Electrostatic and van der Waals cutoffs were set to 0.9 and $1.4 \mathrm{~nm}$, respectively. The Particle Mesh Ewald (PME) algorithm [32] was used for long range electrostatic interactions.

Following equilibration, full molecular dynamics simulations were executed for $100 \mathrm{~ns}$, with coordinates written at 2 ps intervals. GROMACS routine utility scripts were exploited to analyze the simulation trajectories. Structure stability was assessed by the parameters of root mean square deviation (RMSD), radius of gyration $\left(\mathrm{R}_{\mathrm{g}}\right)$, root mean square fluctuation (RMSF), time evolution of hydrogen bond number, and secondary structure. RMS fluctuations of protein, $\mathrm{C} \alpha$ atoms, and backbone atoms observed in all five models were averaged for analysis. RMS fluctuations of backbone atoms were mapped onto backbone ribbon structures, and distributed among three categories: 1) lower amplitude atomic motions of backbone residues for which RMSF values were below the average RMSF ( $\mathrm{RMSF}_{\mathrm{avg}}$ ); 2) intermediate amplitude atomic motions of backbone residues for which RMSF values exceeded $\mathrm{RMSF}_{\text {avg }}$ up to a value $50 \%$ higher than $\mathrm{RMSF}_{\text {avg, }}$ i.e. (1.5 x RMSF ${ }_{\text {avg }}$ ); 3) higher amplitude atomic motions of backbone residues for which RMSF values exceeded those in category 2, i.e. $>1.5 \mathrm{x} \mathrm{RMSF}_{\text {avg }}$ ). Data were visualized using xmgrace (http:// plasma-gate.weizmann.ac.il/Grace/) and gnuplot v 4.6 (http://www.gnuplot.info/). All structure models were visualized and aligned using MolMol [33], VMD [34], and PyMOL (Molecular Graphics System, v1.5.0.4 Schrödinger, LLC). Figures were prepared in PyMOL and gnuplot.

\section{Results}

Molecular dynamics was performed on the STAS domains of rat prestin and human pendrin. The sequence of the rat prestin STAS construct used to perform loop L5 modeling is shown in Figure 1A. Also shown is the aligned sequence of human pendrin STAS domain. The five best loop L5-modeled crystal structures (Fig. 1B) superposed well on the backbone atoms of the PDB ID 3LLO rat prestin STAS domain parent structure within 0.16-0.19 RMSD, consistent with preservation of the structural fold and secondary structure packing of the rest of the STAS domain. Fig. 1C shows the overlay of the best of five L5 modeled rat prestin STAS crystal structures with its parent structure. Good stereochemical quality of the loop L5-modeled crystal structures is evident in the Ramachandran map (not shown) for the best five structural models (Fig. 1B).

The best of the five L5 loop-modeled structures (Fig. 1C) served as template for modeling of the 143 aa human pendrin STAS domain lacking its IVS. The five best modeled pendrin 


\section{(A)}

$$
\begin{aligned}
& \text { rpres 505-SPSYTVLGQLPDTDVYIDI DAYEEVKEIPGIKIFQINAPIYYANSDLYSSALKRKTGVNGSENIHTVILD-645 } \\
& \text { hpen 515-FPSWNGLGSIPSTDIYKSTKNYKNIEEPQGVKILRFSSPIFYGNVDGFKKCIKSTVGFDGSVPIHSLVLD-661 } \\
& \text { 646-FTQVNFMDSVGVKTLAGIVKEYGDVGIYVYLAGCSAQVVNDLTSNRFFENPALKELLFHSIHDAVLGQVREA-718 } \\
& \text { 662-CGAISFLDVVGVRSLRVIVKEFQRIDVNVYFASLQDYVIEKLEQCGFFDDIRKDTFFLTVHDAILYLQNQVK-734 }
\end{aligned}
$$

\begin{tabular}{|c|c|c|c|c|c|c|c|c|c|c|}
\hline \multirow{6}{*}{$\begin{array}{l}\text { Residues in regions... } \\
\text { most favored } \\
\text { additionally allowed } \\
\text { generously allowed } \\
\text { disallowed }\end{array}$} & \multicolumn{5}{|c|}{ rat prestin models } & \multicolumn{5}{|c|}{ human pendrin models } \\
\hline & 1 & 2 & 3 & 4 & 5 & 1 & 2 & 3 & 4 & 5 \\
\hline & & & 952 & & 980 & & & & & 052 \\
\hline & $\begin{array}{r}95.2 \\
4.8\end{array}$ & $\begin{array}{r}96.0 \\
4.0\end{array}$ & $\begin{array}{r}95.2 \\
4.8\end{array}$ & $\begin{array}{r}94.4 \\
4.8\end{array}$ & $\begin{array}{r}96.0 \\
40\end{array}$ & $\begin{array}{r}96.0 \\
32\end{array}$ & $\begin{array}{r}96.0 \\
4.0\end{array}$ & $\begin{array}{r}94.4 \\
40\end{array}$ & $\begin{array}{r}94.4 \\
5.6\end{array}$ & $\begin{array}{r}95.2 \\
4.0\end{array}$ \\
\hline & 0.0 & 0.0 & 0.0 & 0.8 & 0.0 & 0.0 & 0.0 & 0.8 & 0.0 & 0.0 \\
\hline & 0.0 & 0.0 & 0.0 & 0.0 & 0.0 & 0.8 & 0.0 & 0.8 & 0.0 & 0.8 \\
\hline \multicolumn{11}{|c|}{$\begin{array}{l}\text { Close Contacts and Deviations from Ideal Geometry } \\
\text { (from PDB validation software)... }\end{array}$} \\
\hline Number of close contacts (within 2.2 A): & 0 & 0 & 0 & 0 & 0 & 0 & 0 & 0 & 0 & 0 \\
\hline RMS deviation for bond angles (degree) & 2.1 & 2.2 & 2.3 & 2.2 & 2.2 & 2.2 & 2.2 & 2.3 & 2.3 & 2.3 \\
\hline RMS deviation for bond lengths $(A)$ : & 0.019 & 0.019 & 0.019 & 0.019 & 0.019 & 0.020 & 0.019 & 0.019 & 0.019 & 0.020 \\
\hline
\end{tabular}

(B)

(C)

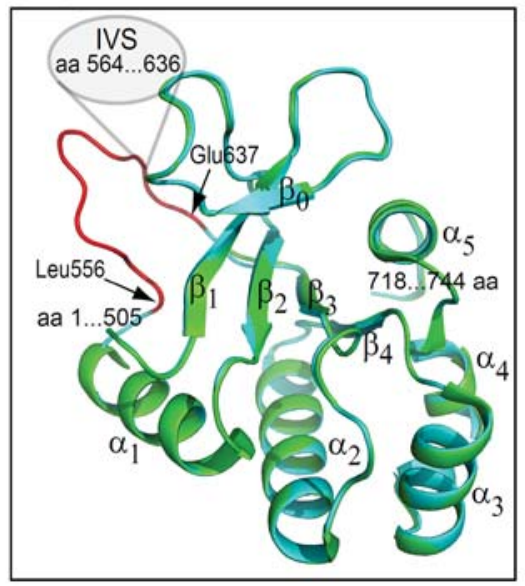

(D)

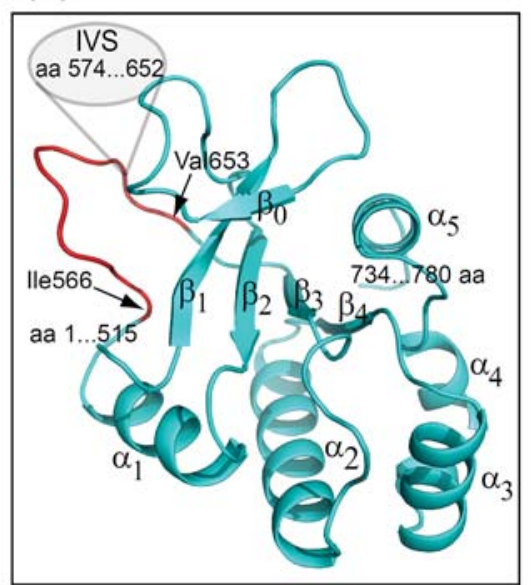

Fig. 1. STAS domain backbone structures of rat prestin and human pendrin. (A) Amino acid sequence alignment of STAS domains of rat prestin (rpres) and human pendrin ( $h$ pen), with secondary structure notations of loops (L), $\alpha$-helices $(\alpha)$, and $\beta$-strands $(\beta)$ [11]. The rat prestin STAS domain sequence encompasses aa 505-718, excluding the IVS region of aa 564-636 (between helix $\alpha 1$ and strand $\beta 3$ ). The sequence used for modeling includes 11 loop residues within L5 previously undetected in the crystal structure \{556-LeuLysArgLysThrGlyValAsn-563, a GlySer linker pair introduced by cloning (shown in italics), and Glu-637\}. The human pendrin STAS sequence encompasses aa 515-734, excluding the intervening sequence (IVS) of aa 574-652 (also between helix $\alpha 1$ and strand $\beta 3$ ). The pendrin sequence includes 11 loop residues within L5 \{566-IleLysSerThrValGlyPheAsp-573, a GlySer pair introduced by cloning (shown in italics), and Val653\} aligned with the corresponding residues modeled in rat prestin STAS. (B) Ramachandran plots of STAS domains of rat prestin and human pendrin showing the stereochemical quality of backbone dihedral angles $(\phi, \psi)$. Each of the five best model structures of STAS domain of rat prestin and of human pendrin incorporates the modeled eleven loop residues absent from the prestin crystal structure (see text for details). The loop-modeled best STAS structure of rat prestin (with low dope score and best $(\phi, \psi)$ score on Ramachandran plot) served as template to model the STAS domain structure of human pendrin. (C) Superposition of the loop-modeled structure (cyan) and crystal structure (green, PDB 3LLO) of rat prestin STAS domain encompassing aa 505-718 (excluding the IVS). Shown in red is the modeled conformation of the 11 loop residues undetected in the crystal structure. (D) The modeled STAS domain structure of human pendrin (cyan) encompassing aa 515-734 (excluding the IVS). Red denotes the conformation of the 11 loop residues. 
Fig. 2. Radius of gyration, $\mathrm{R}_{\mathrm{g}}(\mathrm{nm})$, experienced by backbone atoms of the STAS domains of (A) rat prestin and (B) human pendrin during 100 ns of molecular dynamics simulation. Inset shows root mean square deviation (RMSD) experienced by the STAS domain backbone atoms relative to the starting structures during the $100 \mathrm{~ns}$ simulation trajectory.

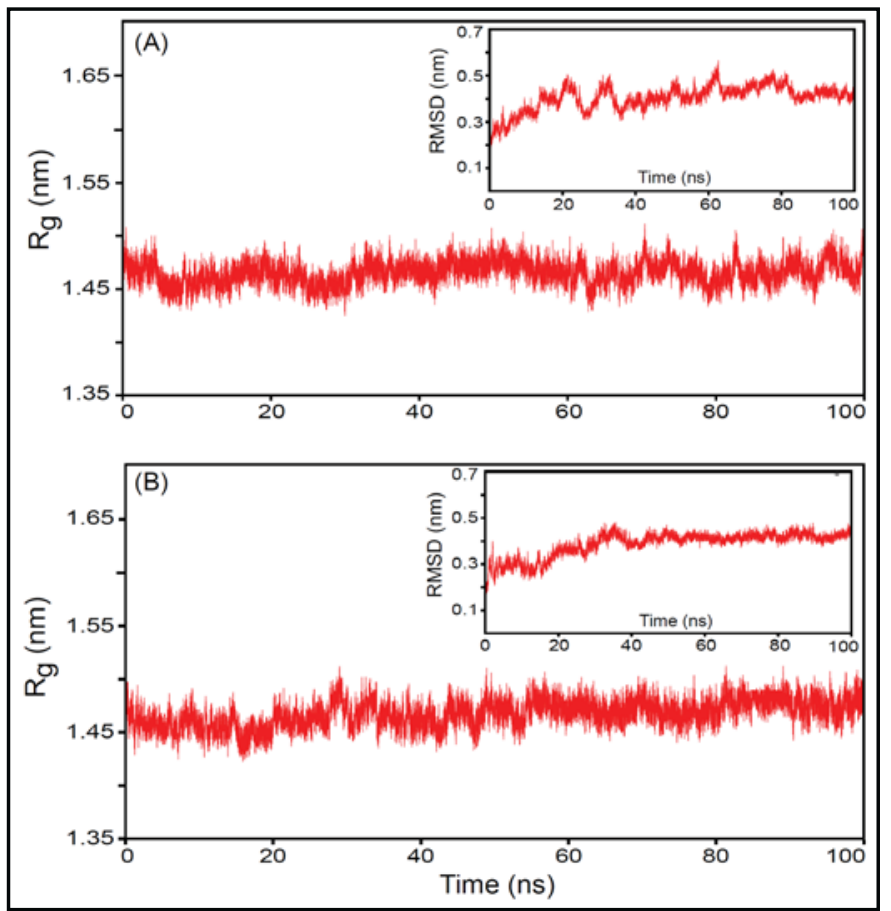

STAS structures showed good stereochemical quality (Fig. 1B). Dihedral statistics of energyminimized best models revealed a high number of residues occupying favored regions in the Ramachandran map (not shown), with no residues of either STAS domain structure in disallowed regions. Fig. 1D presents the best of the five modeled pendrin STAS structures.

The structural models obtained by loop-modeling of the rat prestin STAS crystal structure and by homology modeling of the human pendrin STAS domain exhibit secondary structured elements of generally similar length, as viewed in MOLMOL, with minor differences. $\beta$-strand lengths in all STAS models were identical to those of the prestin STAS crystal structure. However, the following model-dependent differences in helical lengths are noted: helix $\alpha 1$ is extended by two C-terminal residues in one of the five rat prestin models and is shortened by one $\mathrm{N}$-terminal residue in one of the five human pendrin models, as compared to the crystal structure. Helix $\alpha 2$ is extended by one C-terminal residue in one of the five prestin models and in one of the five pendrin models. In addition, two of the five pendrin STAS models exhibit extension of helix $\alpha 5$ by two C-terminal residues.

Figure 2 shows the radius of gyration $\left(R_{g}\right)$ of the simulation profile of the best of the five models for the STAS domains of rat prestin and human pendrin. Average $\mathrm{R}_{\mathrm{g}}$ values are 1.46 $\mathrm{nm}$ for prestin STAS (Fig. 2A) and $1.47 \mathrm{~nm}$ for pendrin STAS (Fig. 2B). The data suggest that structural conformations of both STAS domains behave similarly during the simulation. The structural folds stabilize within the first 20 ns and remain almost constant over the next 60 $\mathrm{ns}\left(\mathrm{R}_{\mathrm{g}}\right.$ value stability during the simulation trajectory indicates stability of protein folding and conformational state). The final 5 ns of the simulations show transient variation in $R_{g}$ values in both STAS domain structures, probably reflecting minor conformational changes. Simulation data on the other four of five best structural models show similar $\mathrm{R}_{\mathrm{g}}$ distribution patterns between 1.41-1.53 nm for all STAS domain models of both rat prestin and human pendrin. These results suggest that the modeled rat prestin STAS domain may be marginally more stable than that of human pendrin during the $100 \mathrm{~ns}$ simulation run. The modest variations in $\mathrm{R}_{\mathrm{g}}$ profile throughout the simulation may reflect conformational readjustments accompanying $\alpha 1-\alpha 2$ inter-helical re-packing (Fig. 2). These results corroborate the RMSD and atomic fluctuation results observed in localized regions of the STAS domains (see below).

In Figure 2, the inset panels present the evolution of RMS deviation (RMSD) in the best STAS domain models of rat prestin and human pendrin, from starting structures until the end of the simulation period. The initial RMSD $_{\text {avg }}$ values for the modeled STAS domains 
Fig. 3. Overlay of backbone ribbon structures of STAS domains of (A) rat prestin and (B) human pendrin before (cyan) and after simulation (purpleblue). The modeled loop regions absent from the prestin crystal structure are rendered in wheat (before simulation) and red (after simulation). Structures were superposed by aligning backbone atoms $\left(\mathrm{N}, \mathrm{C}^{\alpha}\right.$, and $\left.\mathrm{C}^{\prime}\right)$ in PyMOL. Figures are oriented to highlight the localized conformational changes within the inter-helical $\alpha 1-\alpha 2$ region. Average backbone RMSD of each residue of the modeled STAS domains from (C) rat prestin and (D) human pendrin, plotted as a function of residue number. RMSD values were averaged from the 5 chosen models of rat prestin STAS and human pendrin STAS. Secondary structure is at top (Figs. C\&D).
(A)

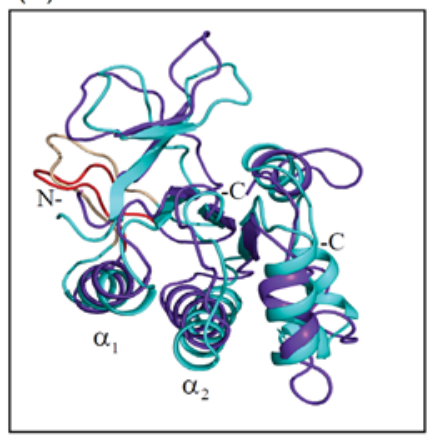

(C)

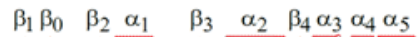

(B)

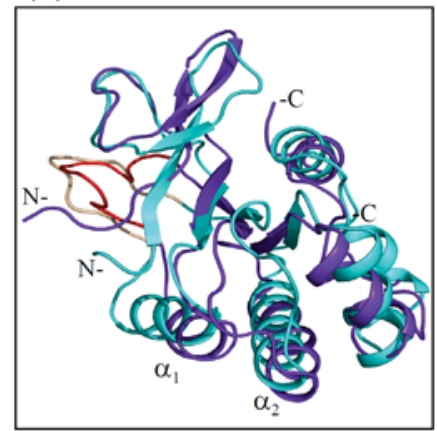

(D)

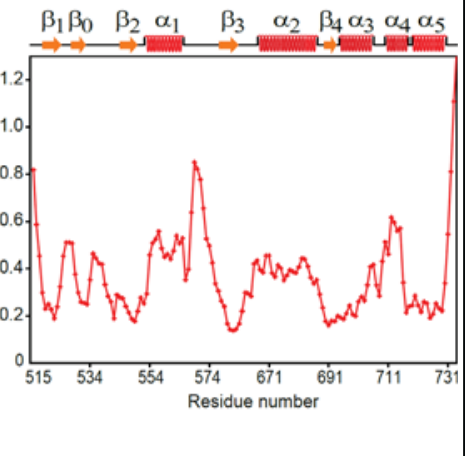

of rat prestin and human pendrin are $0.40 \mathrm{~nm}$ and $0.39 \mathrm{~nm}$, respectively. $\mathrm{RMSD}_{\text {avg }}$ values progressively increase with increasing simulation time. The consistent increase in RMSD of both modeled proteins during the initial 40 ns likely reflects conformational rearrangements adjusting structural restraints and non-covalent intramolecular interactions. RMSD increase during the following $60 \mathrm{~ns}$ is modest for both modeled proteins, with transient increases noted for rat prestin STAS during the intervals at 58-63 ns and 71-80 ns. The RMSD ${ }_{\text {avg }}$ values during the final $60 \mathrm{~ns}$ of simulation are $0.43 \mathrm{~nm}$ for rat prestin STAS and $0.42 \mathrm{~nm}$ for human pendrin STAS. The data suggest that structural RMSD values remain stable after initial equilibration, reflecting overall conformational stability of both STAS domains.

Overlay of the STAS domain backbone atom ribbon structures before ( $0 \mathrm{~ns})$ and after 100 ns dynamics simulation of the best models shows the pre-post simulation RMSD of 3.19 $\AA$ for rat prestin STAS and $3.40 \AA$ for human pendrin STAS (Fig. 3A\&B). The pre-minus-post simulation averaged RMSD of all 5 STAS models is $3.42 \AA$ for rat prestin and $3.71 \AA$ for human pendrin. The results reveal the loop regions to be relatively more flexible and exhibiting greater deviations from their initial structures than $\alpha$-helices and $\beta$-strands. Analyses of averaged RMSD data show that the largest backbone atom RMSD difference values (across the simulation time period) among the loop regions of human pendrin STAS include L1 (6.2 $\AA)$, L2 (4.5 $\AA$ ), the modeled (and longest) loop L5 (5.0 $)$, L9 (4.1 $)$, and L11 (7.3 $\AA$ ). Those of rat prestin STAS include L1 (4.1 $)$ ), L2 (4.0 $)$, L5 (4.6 ̊), L9 (4.4 $)$ ), and L11 (7.6 $)$. In both modeled proteins, the C-terminal loop L11 exhibits larger deviations than does N-terminal loop L1. RMSD of modeled loop L5 (lacking the IVS) exceeds that of any loop other than the C-terminal loop L11 and (only in human pendrin STAS) the N-terminal loop L1. Consistent with the higher overall RMSD values of human pendrin STAS (Fig. 2 and see above) most secondary structured segments of human pendrin STAS exhibit RMSD values larger than the corresponding values of rat prestin STAS, except for L3, $\alpha 3, \alpha 5$, and L7-L11, and $\beta 5$ (Fig. 3C\&D).

Helices $\alpha 1, \alpha 2$, and $\alpha 4$ show larger deviations (Fig. 3), especially in or near the C-terminal regions of helices $\alpha 1$ and $\alpha 2$. Other secondary structured elements show more 


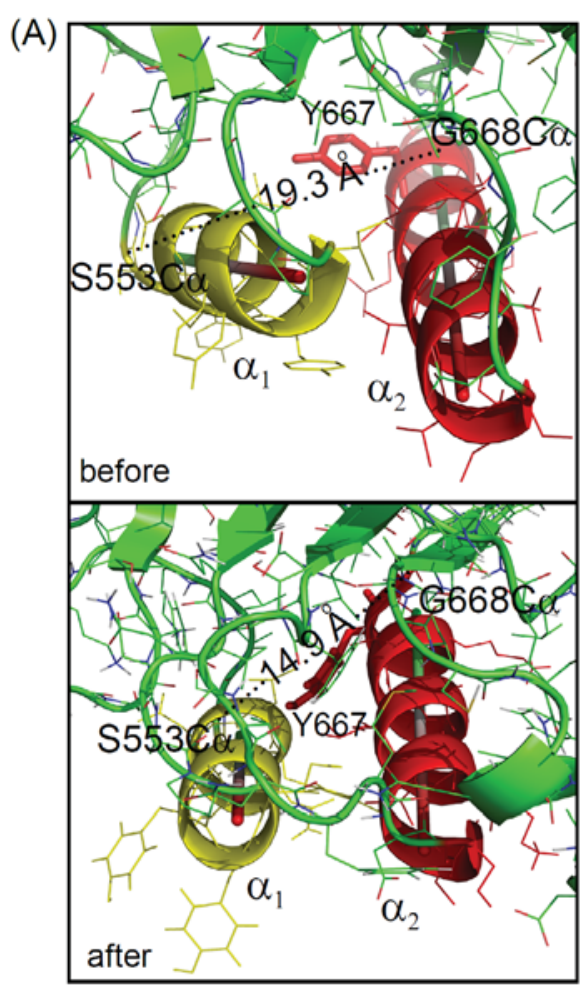

(B)

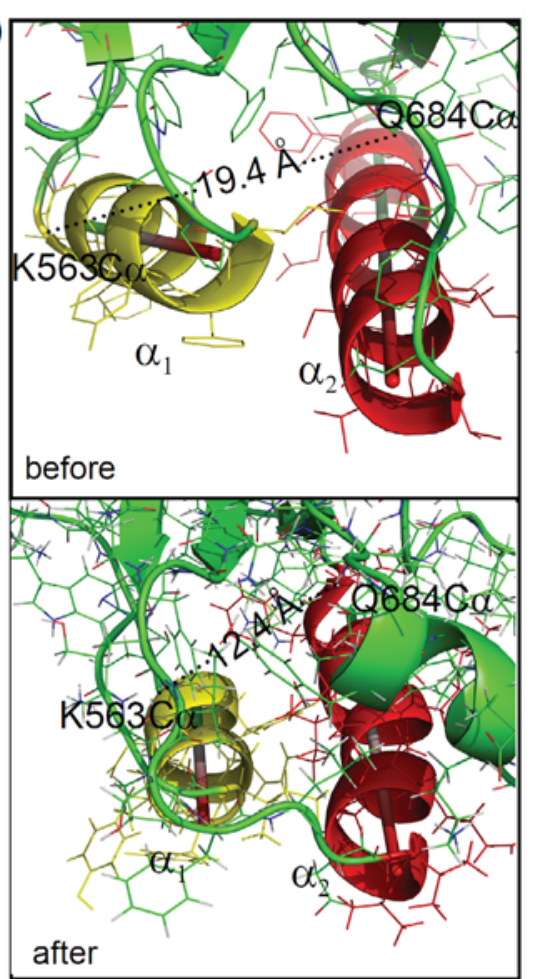

(C)
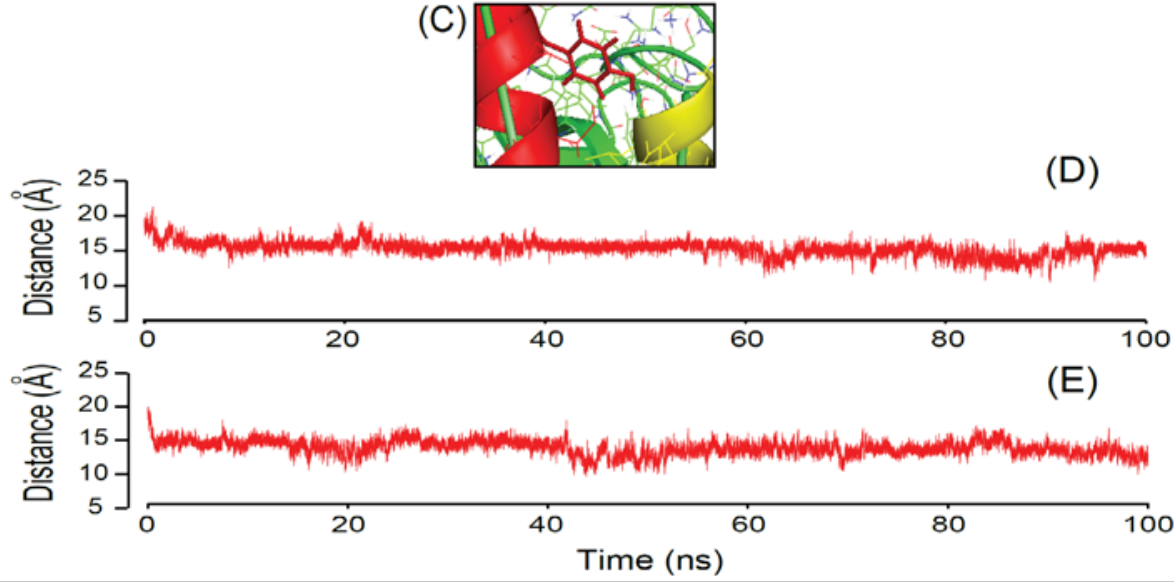

Fig. 4. Stereo-view of loop-modeled and energy-minimized STAS domain backbone ribbon structures of (A) rat prestin and (B) human pendrin, highlighting the conformational changes in relative orientation of helices $\alpha_{1}$ (yellow) and $\alpha_{2}$ (red) before (upper panels) and after $100 \mathrm{~ns}$ dynamics simulations (lower panels). (C) Enlarged stereo-view of Tyr667 ring within the loop-modeled and energy-minimized STAS domain backbone ribbon structure of rat prestin after $100 \mathrm{~ns}$ dynamics simulations (color scheme as in (A), bottom panel). (D) Simulation time-dependent distance between $\mathrm{C}^{\alpha}$ atoms of S553 and G668 in rat prestin STAS. (E) Simulation time-dependent distance between $\mathrm{C}^{\alpha}$ atoms of $\mathrm{K} 563$ and Q684 in human pendrin STAS.

modest deviations. Interestingly, the post-simulation deviation of the $8 \mathrm{C}$-terminal aa of rat prestin STAS helix $\alpha 2(3.4 \AA)$ considerably exceeds that of its $9 \mathrm{~N}$-terminal aa $(2.1 \AA ̊)$. The simulation trajectory reveals a significant time-dependent change in the relative orientation of helices $\alpha 1$ and $\alpha 2$, manifesting predominantly in the approximation and repacking of both helical C-termini, and reflected in altered $\mathrm{C} \alpha$-C $\alpha$ distances between the $\mathrm{C}$-terminal residues of helices $\alpha 1$ and $\alpha 2$ (Fig. 4). 


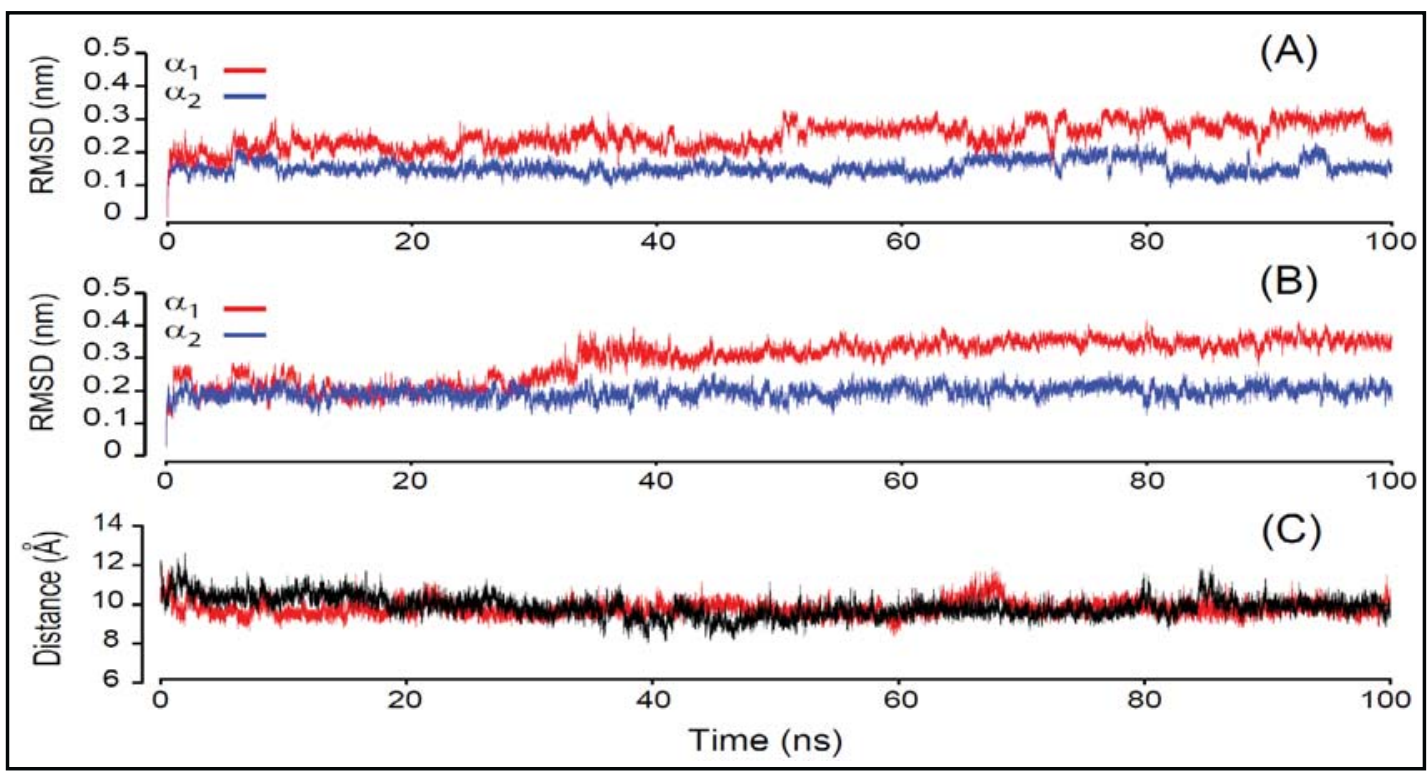

Fig. 5. Evolution of RMSD values for $\mathrm{C}^{\alpha}$ group of atoms of helices $\alpha_{1}$ and $\alpha_{2}$ from the simulation of STAS domains of (A) rat prestin and (B) human pendrin during 100 ns simulation. (C) $\alpha_{1}-\alpha_{2}$ inter-helical distance trajectory for $\mathrm{C}^{\alpha}$ group of atoms in STAS domains of rat prestin (red) and human pendrin (black) during 100 ns simulation.

The simulation data on the best model of rat prestin STAS show that the initial $C \alpha-C \alpha$ distance $\left(\mathrm{d}_{\mathrm{C} \alpha-\mathrm{C \alpha}}\right)$ of $19.34 \AA$ between the extreme C-terminal residues of helices $\alpha 1$ (Ser553) and of $\alpha 2$ (Gly668) (19.67 $\AA$ in the pdb 3LLO crystal structure) drops to a post-simulation value of $14.92 \AA$ (Fig. 4A). Similarly, the initial C $\alpha$-C $\alpha$ distance of $19.42 \AA$ in human pendrin STAS between the extreme C-terminal residues of helices $\alpha 1$ (Lys563) and of $\alpha 2$ (Gln684) falls to a post-simulation value of $12.36 \AA$ (Fig. 4B). Shown in Fig. 4C is the enlarged and rotated view of post-simulation backbone conformation of rat prestin (as shown in Fig. 4A) illustrating the packing mode of Tyr667 ring. The substantial reductions in $\mathrm{d}_{\mathrm{C \alpha}-\mathrm{C \alpha}}$ (Figs. $4 A \& B)$ reflect altered conformation of the $\alpha 1-\alpha 2$ helical pair in both STAS domains, as shown in Fig. 4D for the temporal evolution of $\mathrm{d}_{\mathrm{C \alpha}-\mathrm{C} \alpha}$ between residues $553(\alpha 1)$ and $668(\alpha 2)$ of rat prestin STAS, and in Fig. 4E between residues $563(\alpha 1)$ and $684(\alpha 2)$ of human pendrin STAS. Initiation of the simulation period leads abruptly (within $2 \mathrm{~ns}$ ) to $\mathrm{d}_{\mathrm{C \alpha}-\mathrm{C \alpha}}$ reductions to the overall average values (15.3 $\AA$ for prestin, $13.9 \AA$ for pendrin). Although $d_{C \alpha-C \alpha}$ varies during the $100 \mathrm{~ns}$ simulation (especially in human pendrin STAS), it never regains the initial, more elevated values. The intermediate trajectory structural folds suggest that the reduction in $\mathrm{d}_{\mathrm{C \alpha}-\mathrm{C} \alpha}$ is not a localized, step-wise conformational change, but rather a gradual one, apparently coupled to substantial conformational changes in other structured regions that might contribute to the approximation of helices $\alpha 1$ and $\alpha 2$.

The time evolution of RMSD in helices $\alpha 1$ and $\alpha 2$ is analyzed in Fig. 5A\&B. The average initial RMSD values for these two helices are $2.5 \AA$ and $1.5 \AA$ for rat prestin STAS and and 2.9 $\AA$ and $1.9 \AA$ for human pendrin STAS. The change in $C \alpha-C \alpha$ distance $\left(d_{C \alpha-C \alpha}\right)$ between helices $\alpha 1$ and $\alpha 2$ plotted vs. simulation time (Fig. 5C) reveals average values of $9.8 \AA$ for rat prestin and $9.9 \AA$ for human pendrin. The initial distance falls to the average values within $5 \mathrm{~ns}$ in both STAS domains, more rapidly in rat prestin than in human pendrin. The variation over time in $d_{C \alpha-\alpha \alpha}$ is slightly larger in human pendrin STAS than in rat prestin STAS, but never regains the initial value. That distance in human pendrin STAS gradually decreases over the first $40 \mathrm{~ns}$, drops modestly for the next $12 \mathrm{~ns}$, then increases marginally and remains nearly constant until end-simulation. These results are consistent with the data above showing that most of the $\alpha 1-\alpha 2$ inter-helical re-orientation takes place early during the simulation, and 


\section{Cellular Physiology and Biochemistry}

Cell Physiol Biochem 2014;33:605-620

\begin{tabular}{l|l}
\hline DOI: $10.1159 / 000358638$ & (c) 2014 S. Karger AG, Basel
\end{tabular}

www.karger.com/cpb

Sharma/Zelikovic/Alper: Molecular Dynamics Simulations of Mammalian STAS Domains

that the rearranged helical packing is sustained throughout the remainder of the $100 \mathrm{~ns}$ simulation period.

The $\alpha 1-\alpha 2$ inter-helical packing in rat prestin STAS exhibits greater separation of the helical C-termini than is evident in either the STAS domains of Rv1739c and ychM, or in the much larger number of anti-sigma factor antagonist structures. Dynamics simulations of rat prestin STAS led to substantial conformational changes in and near the C-termini of helices $\alpha 1$ and $\alpha 2$. The simulations showed that helices $\alpha 1$ and $\alpha 2$ dynamically approach each other during the simulation trajectory, and their positions are relatively less rigid than those of other helices and strands in the structure. The dynamic rearrangement observed during the simulation significantly reduced the $\alpha 1-\alpha 2$ inter-helical angle in rat prestin STAS from the initial value of $38.7^{\circ}$ to $21.1^{\circ}$ post-simulation (measurements based on axes extrapolated towards the helical N-termini). The $\alpha 1-\alpha 2$ inter-helical angle in the modeled human pendrin STAS domain decreases substantially from its initial value of $37.7^{\circ}$ to a post-simulation value of $16.3^{\circ}$ (Fig. 4A\&B). The angular flexibility between helices $\alpha 1$ and $\alpha 2$ is reflected in the continuous conformational changes evident during the intermediate stages of the simulation trajectory. These data supporting the presence of dynamic conformational fluctuations suggest that the published rat prestin crystal structure may represent one of several possible solution conformations. Although our $100 \mathrm{~ns}$ simulation time captures significant changes in inter-helical structure, still longer run times could provide additional details on this interhelical repacking. The post-simulation $\alpha 1-\alpha 2$ inter-helical packing in the modeled STAS domains of rat prestin and human pendrin more closely resembles that observed in other available STAS domain structures, with $\alpha 1-\alpha 2$ inter-helical angles of $<30^{\circ}$. Structures of antisigma factor antagonists display still smaller inter-helical angles verging on parallel (as low as $2.80^{\circ}$ for the average NMR structure of SpoIIAA).

Structures extracted from the simulation trajectory at multiple times reveal that helices $\alpha 1$ and $\alpha 2$ both undergo conformational changes throughout the simulation. The phenol ring of residue Tyr667 also shows clear orientational differences throughout the simulation trajectory. Its frequent flips exploit nearby changes in helical conformation to sample multiple orientations in conformational space for energy minimization of the $\alpha 1$ $\alpha 2$ core region. In separate simulation experiments with STAS domains of rat prestin and human pendrin, the approximation of helices $\alpha 1$ and $\alpha 2$ is coupled with a modest axial shift of helix $\alpha 1$ towards its $\mathrm{N}$-terminus, allowing stereo space sufficient for the phenol ring of Tyr 667 to pack between helices $\alpha 1$ and $\alpha 2$. Trajectory analyses of human pendrin STAS suggested that the phenyl ring of corresponding residue Phe683 is equally dynamic, exhibiting conformational changes similar to those rat prestin Tyr667. As in rat prestin STAS, helices $\alpha 1$ and $\alpha 2$ of human pendrin STAS exhibited inter-helical approximation of their C-terminal regions and facilitated packing of the Phe683 phenyl ring. These structural changes accompany conformational changes in other structural regions. Taken together, the STAS domain MD results suggest that localized conformational changes at any given time are occuring in the context of overall structural rearrangements.

Secondary structural elements are generally preserved during the simulation, with retention of their folded states (data not shown). Conformational change was more likely in loops and helices, whereas strands showed greater rigidity and, generally, did not experience significant conformational changes. Helices $\alpha 1$ and $\alpha 4$ of human pendrin STAS and helix $\alpha 4$ of rat prestin STAS undergo conformational sampling and rearrangement during the $100 \mathrm{~ns}$ simulation. Disappearance of helix $\alpha 4$ in the final structure is noted in several models, although it reappears transiently at several stages during the run. $\alpha 1$ helical length undergoes transient shortening by 2 residues at its $\mathrm{N}$-terminus in 3 of the 5 human pendrin STAS models. $\alpha 2$ helical length is transiently shortened by 1 residue at its $\mathrm{C}$-terminus in 2 of the 5 models in STAS domains of both proteins. These changes in the protein conformation are not un-anticipated in the context of re-orientation of the helices $\alpha 1$ and $\alpha 2$ of the core region of protein structure. Indeed, comparison of the 3LLO prestin STAS crystal structure with structural predictions deduced from the corresponding NMR chemical shift data ([11]) highlights comparable differences in lengths of multiple helices and strands. (Note 


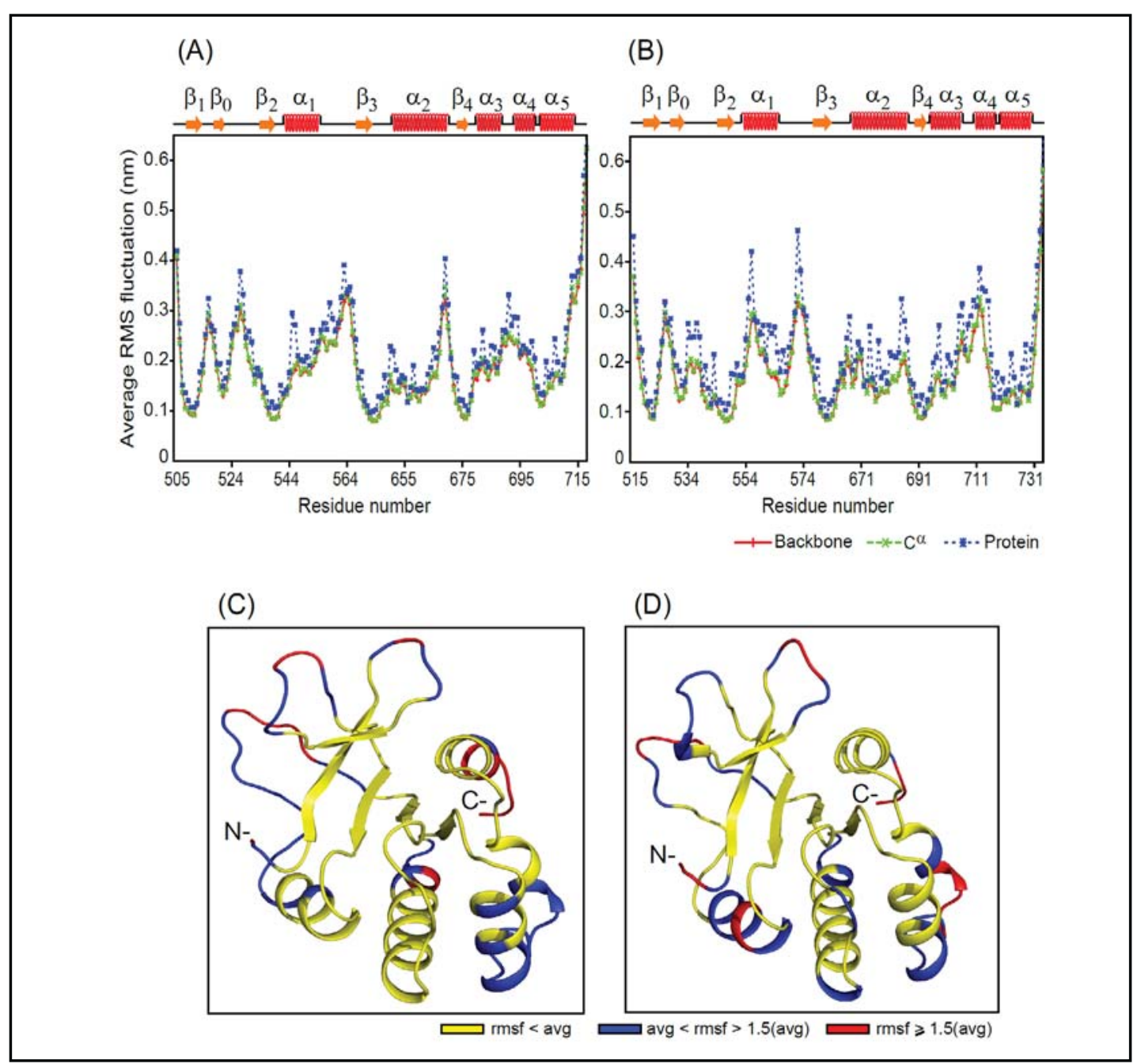

Fig. 6. Atom-by-atom root mean square fluctuations (RMSF) of STAS domains of (A) rat prestin and (B) human pendrin, plotted as functions of residue number for $\mathrm{C}^{\alpha}$ atoms (green), backbone atoms (red), and all protein atoms (blue). Data are shown for the average RMSF values of all five models for each STAS domain of rat prestin and human pendrin. Higher RMSF values indicate larger atomic motions (Secondary structures are above panels $A$ and $B$ ). RMSF heat maps of STAS domain average RMSF values (RMSF ${ }_{\text {avg }}$ ) for backbone atoms of all five models of (C) rat prestin and (D) human pendrin (scale below panels). Backbone RMSF values for entire STAS domains of rat prestin and human pendrin are 0.19 and $0.17 \mathrm{~nm}$, respectively (See text for details).

however, that the crystallized construct lacked $9 \mathrm{C}$-terminal amino acid residues present in the construct analyzed by NMR.) Our simulation data suggest that overall conformational plasticity is coupled with cooperative stereo-adjustment among secondary structured elements. High stereo-chemical quality of these structures was maintained throughout the 100 ns simulation, with transient entry of only 3 or 4 residues into disallowed regions (none from helices $\alpha 1$ and $\alpha 2$ ), and with heavy atoms approximating no closer than $2.2 \AA$.

Figure 6A\&B show atomic RMS fluctuations (RMSF) in the three categories of $\mathrm{C} \alpha$ atoms, backbone atoms ( $\left.\mathrm{N}, \mathrm{C} \alpha, \mathrm{C}^{\prime}\right)$, and all protein atoms (see Materials and Methods), plotted as functions of STAS residue number for both rat prestin and human pendrin. The RMSF distribution profiles are similar for all three categories, but fluctuation amplitudes for all protein atoms are higher than for other categories, reflecting higher degrees of freedom. RMSF values are generally higher for loop residues than those in helices and strands of 


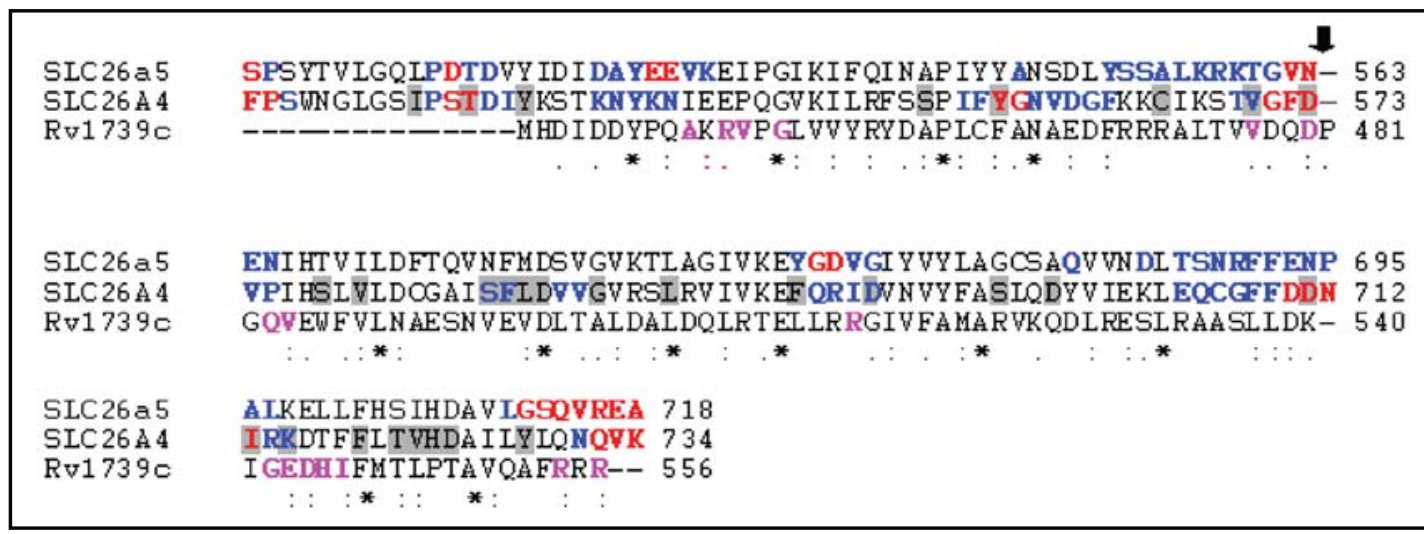

Fig. 7. Amino acid sequence alignment (ClustalW 2.1) of STAS domains of rat prestin (SLC26a5), human pendrin (SLC26A4), and M. tuberculosis Rv1739c. Amino acids are highlighted based on their backbone atoms average RMSF values of all five models (Fig. 6A\&B). For prestin, blue indicates RMSF between 0.19 and 0.28 , and red indicates RMSF $\geq 0.28$. For prestin, blue indicates RMSF between 0.17 and 0.26 , while red indicates $\geq 0.26$ (see text for details). Rv1739c STAS residues in pink exhibit chemical shift perturbation upon GDP binding [9]. Pendrin residues in gray blocks are loci of human disease-associated missense mutations. Amino acid residue numbers (at right) are those of the full-length proteins. The black arrow indicates positions of the IVS regions excised from the mammalian sequences (see Figure 1 for details). Asterisks (below) denote completely conserved residues.

greater rigidity. $\mathrm{RMSF}_{\text {avg }}$ values of secondary structured regions in the 5 best individual models follow similar trends. Several secondary structured regions show higher-than-overall $\mathrm{RMSF}_{\text {avg }}$ values for backbone atoms. RMSF avg of the mean values of the 5 best models suggest that L1, L2, L3, L5, L7, L9, and L11 in rat prestin STAS and L1, L2, L5, L6, L9, L11, $\alpha 1$, and $\alpha 4$ in human pendrin STAS exhibit higher-than-overall backbone atom $\mathrm{RMSF}_{\text {avg }^{\prime}} \mathrm{C}$-terminal loop L11 of rat prestin shows atomic motions 2-fold higher in amplitude than $\mathrm{N}$-terminal loop L1. Both L5 loops show greater atomic motion than all other secondary structures except for terminal loops, and (in human pendrin STAS only) regions $\alpha 1, \alpha 4$, and L2. These results corroborate the corresponding RMSD data (see above). STAS domain $\beta$-strand RMSF values are similar in rat prestin and human pendrin. To understand local fluctuations, individual STAS domain residues exhibiting higher-than-average atomic motions were identified in all three structural categories (see Materials and Methods). Fig. 6C\&D map these RMSF values in pseudocolor onto the backbone ribbon structure to highlight those residues exhibiting lower and higher amplitude atomic motions (Fig. 6C\&D). Several of the residues exhibiting higher-than-average motional amplitude are identical in rat prestin and human pendrin. The majority of residues exhibiting higher-than-average motional amplitude belong to loop regions or to the termini of helices and strands (Exceptions include rat prestin STAS helix $\alpha 3$ and human pendrin STAS helices $\alpha 1$ and $\alpha 4$ ).

The STAS domain of M. tuberculosis Rv1739c is the only SulP structure in which small molecule ligand-binding residues have been identified [9]. Alignment of the Rv1739c STAS domain amino acid sequence with STAS domain residues of rat prestin and human pendrin allows comparison of the Rv1739c residues chemically shifted by GDP [including L3 (immediately preceding $\beta 2$; L5 (immediately preceding $\beta 3$ ); helix-loop region ( $\alpha 2$-L7); helix $\alpha 4$; and C-terminal loop L11] with STAS residues of prestin and pendrin exhibiting elevated backbone atom motions (Fig. 7). Among the 28 pendrin residues known as sites of human disease-associated missense mutations, Val570, Asp573, Ile713 (conserved in both sequences), and Lys715 exhibit both increased atomic motions and alignment with Rv1739c STAS residues located in or near regions of GDP-binding (Fig. 7).

Charge reversal mutations of two regions of clustered charge within the nearly identical STAS domain of gerbil prestin have been shown to modify the non-linear capacitance (NLC) 


\section{Cellular Physiology and Biochemistry}

Cell Physiol Biochem 2014;33:605-620

DOI: $10.1159 / 000358638$

Published online: February 27, 2014

c) 2014 S. Karger AG, Basel

www.karger.com/cpb

617

Sharma/Zelikovic/Alper: Molecular Dynamics Simulations of Mammalian STAS Domains

of the prestin transmembrane domain [35]. One charged cluster in the IVS is absent from the prestin crystal and NMR structures, as well as from our structural models. However, a second charged cluster encompassing rat prestin aa 516-531 includes strand $\beta 0$ flanked by much of L2 on one side and much of L3 on the other. Charge reversal of the Asp and Glu residues in this stretch decreased NLC $Q_{\max }$ and depolarized NLC $V_{h}$ by $40 \mathrm{mV}$, suggesting a likely role for this early portion of the prestin STAS domain in modulating, together with the oppositely charged IVS aa 571-580, the voltage-sensing and charge translocation functions of the prestin transmembrane domain that underlie the unique NLC of prestin. Two deafness-associated missense mutations in pendrin reside within the early STAS domain region corresponding to prestin aa 516-531 (Fig. 7). However, the possible contribution of this STAS domain region to pendrin-mediated electroneutral anion exchange $[36,37]$ remains to be determined.

\section{Discussion}

MD simulations provide atomistic details of the temporal evolution of structural properties crucial to the understanding of protein function [16]. We have reported here MD simulation results of STAS domains of the SLC26 protein rat prestin [11] and the human SLC26 deafness gene product, pendrin [4]. Our study modeled an 11 residue loop, absent from the crystal structure (PDB 3LLO), to obtain a more complete structure of the rat prestin STAS domain (still, however, missing the IVS [11]). The human pendrin STAS domain without its IVS was then modeled on the revised rat prestin STAS model. MD simulations were performed on the five best modeled structures of both rat prestin and human pendrin.

The results of $100 \mathrm{~ns}$ MD simulations suggested that both STAS domain conformations are highly dynamic in solution and likely sample more than one conformation. Dynamic features are evident in the observed conformational motions and the deviations experienced by several structured segments, including (in addition to the terminal loop regions) the four major loop regions L2 (between $\beta 1$ and $\beta 0$ ), L3 (between $\beta 0$ and $\beta 2$ ), L5 (between $\alpha 1$ and $\beta 3$ ), and L9 (between $\alpha 3$ and $\alpha 4$ ), as well as helices $\alpha 1, \alpha 2$, and $\alpha 4$.

The $\alpha 1-\alpha 2$ inter-helical packing in the prestin crystal structure 3LLO [11] is remarkable for its wider separation of the helical C-termini than observed in bacterial STAS structures of the SulP transporters Rv1739c [9] and ychM [7, 12]. The MD simulations predicted $\alpha 1-\alpha 2$ inter-helical dynamics in solution in both prestin and pendrin STAS domains. These helices in pendrin experienced relatively greater atomic motions than in prestin, and underwent greater conformational perturbations throughout the trajectory. This inter-helical plasticity led to significant decreases in $\alpha 1-\alpha 2$ inter-helical angles, resulting in increased similarity to the $\alpha 1-\alpha 2$ packing in structures of other STAS domains and anti-sigma factor antagonists. Coupled with the observation of motions in other structured segments, the $\alpha 1-\alpha 2$ interhelical dynamics suggest that mammalian STAS domains in solution likely sample and adopt multiple conformations. These results defining dynamic protein behavior agree with the reported variation in ${ }^{1} \mathrm{H}^{-15} \mathrm{~N}$ correlation crosspeak intensities in the $2 \mathrm{D}{ }^{1} \mathrm{H}-{ }^{15} \mathrm{~N}$ HSQC spectrum of rat prestin STAS domain [11].

In the crystal structure of rat prestin STAS, the Tyr667 phenol ring (near the C-terminus of helix $\alpha 2$ ) is oriented toward the C-terminus of helix $\alpha 1$. This packing mode of prestin Tyr667 was interpreted as contributing to the separation of the C-termini of helices $\alpha 1$ and $\alpha 2$, preventing parallel packing of these two helices [11]. However, the simulated time evolution data presented in the current study emphasize the dynamic nature of the prestin Tyr667 phenol ring. Although oriented towards helix $\alpha 1$, the phenol ring tilts modestly away from the re-adjusted core region of the $\alpha 1-\alpha 2$ bundle while its circumscribed rotation samples multiple packing modes in all three dimensions (Fig. 4A-C) (see above). The conformational flexibility of the prestin Tyr667 ring was paralleled by substantial deviations of the $\alpha 1-\alpha 2$ inter-helical core region from the crystal structure, consistent with the conformational flexibility of helix $\alpha 1$ and its adjacent L5 loop also evident in the NMR spectra of prestin STAS [11] (see below). 


\section{Cellular Physiology \\ and Biochemistry}

Cell Physiol Biochem 2014;33:605-620

\begin{tabular}{l|l}
\hline DOI: $10.1159 / 000358638$ & (c) 2014 S. Karger AG, Basel
\end{tabular}

www.karger.com/cpb

Sharma/Zelikovic/Alper: Molecular Dynamics Simulations of Mammalian STAS Domains

Crystal packing analysis of the rat prestin crystal structure (PDB 3LLO) reveals in helix $\alpha 1$ (aa 544-553), that only residues Tyr 546 and Tyr 552 make crystal contacts of large surface area. Residues Tyr 545, Ser 549, Asp 550, and Ser553 form modest crystal contacts of very small surface area. The majority of helix $\alpha 1$ (especially its C-terminal region) has free stereo space adequate for dynamics execution, as seen in both our MD study and in the solution NMR data for rat prestin STAS, and consistent with B-factor crystal data for 3LLO [11]. In helix $\alpha 2$ (aa 654-669), residues Lys665, Glu 666, and Asp 669 (located near C-terminus) are involved in forming crystal contacts of larger surface, in contrast to the small surface area of contacts formed by Gly 668. Residues of the $\alpha 2$ helix N-terminal region do not appear to form crystal contacts. Tyr667 is also not involved in forming crystal contacts, such that its phenol ring could change conformation in association with $\alpha 1-\alpha 2$ inter-helical approximation. These observations suggest that select residues in helix $\alpha 2$ could contribute to $\alpha 1-\alpha 2$ inter-helical reorientation during the simulation period, and are also consistent with the undetectable heteronuclear NOE data for several $\alpha 2$ helix residues (Fig. 5 in [11]). The less dynamic features of helix $\alpha 2$ residues in the prestin crystal structure may reflect a more rigid stereo environment in the presence of $\beta$-OG.

As noted by Pasqualletto et al, the absence of NMR assignment of 16 residues (aa 541556) constituting helix $\alpha 1$ and adjacent regions, and 6 residues (aa 649-654) immediately preceding helix $\alpha 2$ (BMRB 16910) of rat prestin STAS was due to resonance broadening likely driven by conformational exchange [11]. These missing assignments, coupled with the heterogeneous distribution of crosspeak intensities of several residues, clearly suggest dynamic behavior of STAS regions. The dynamic features noted by NMR [11] agree with the current MD results, and suggest that the STAS domain fold of rat prestin is dynamic in solution that could sample more than one conformation. Future ${ }^{1} \mathrm{H}^{-15} \mathrm{~N}$ backbone and/or ${ }^{1} \mathrm{H}-{ }^{13} \mathrm{C}$ side-chain NMR relaxation dynamics studies of rat prestin STAS could detect further dynamic features in addition to those predicted by this MD study.

The time-dependent conformational re-arrangement of canonical $\alpha 1-\alpha 2$ inter helical packing observed in STAS domains of rat prestin and human pendrin may be an intrinsic property of all STAS domains of SLC26 and SulP proteins, and of anti-sigma factor antagonists as well. It should be noted that the unique inter-helical packing observed in the rat prestin STAS crystal structure (3LLO) and the results reported in this MD study may reflect, at least in part, the effect of exclusion of the IVS region. In that case the dynamic features noted in the current study should be applicable to future structural studies of SLC26 STAS domain constructs lacking the IVS. However, validation of this speculation awaits comparison with the structure of a complete mammalian STAS domain encompassing the IVS region.

\section{Conclusion}

In conclusion, the current MD study shows that the overall structural fold of the rat prestin STAS domain is stable in solution at $300 \mathrm{~K}$ under the chosen simulation conditions. However, the STAS domain exhibits numerous time-dependent motional alterations, conformational perturbations, and structural fluctuations of defined residues and regions, including plasticity in $\alpha 1-\alpha 2$ inter-helical approximation, features less easily detected (if not undetectable) in crystals [38]. Predicted conformational heterogeneity was of even greater amplitude in the STAS domain of human pendrin. The STAS domain conformational plasticity predicted in both prestin and pendrin, along with the high amplitude motions in pendrin STAS residues associated with deafness mutations that align with GDP binding-residues of Rv1739c [9], contribute to an evolving understanding of STAS domain structure and function in SLC26 anion transporter polypeptides.

\section{Conflicts of Interest}

The authors declare no conflict of interest. 


\section{Cellular Physiology and Biochemistry}

Cell Physiol Biochem 2014;33:605-620

\begin{tabular}{l|l}
\hline DOI: $10.1159 / 000358638$ & (C) 2014 S. Karger AG, Basel
\end{tabular}

www.karger.com/cpb

\section{Acknowledgements}

We thank Dr. Manoj K. Bhasin and Dr. Gabriel Birrane (Harvard Medical School, Beth Israel Deaconess Medical Center) for helpful discussion. Access to Linux PCs on the Orchestra clusters was provided by the Harvard Medical School Computing Facility. This work was supported by the US-Israel Binational Science Foundation grant 2009129 to IZ and SLA, and by the Cell Physiology Core of the Harvard Digestive Diseases Center funded by NIH grant DK34854.

\section{References}

1 Sherman T, Chernova MN, Clark JS, Jiang L, Alper SL, Nehrke K: The abts and sulp families of anion transporters from caenorhabditis elegans. Am J Physiol Cell Physiol 2005;289:C341-351.

-2 Felce J, Saier MH, Jr: Carbonic anhydrases fused to anion transporters of the sulp family: Evidence for a novel type of bicarbonate transporter. J Mol Microbiol Biotechnol 2004;8:169-176.

3 Dorwart MR, Shcheynikov N, Yang D, Muallem S: The solute carrier 26 family of proteins in epithelial ion transport. Physiology (Bethesda) 2008;23:104-114.

4 Alper SL, Sharma AK: The slc26 gene family of anion transporters and channels. Mol Aspects Med 2013;34:494-515.

-5 Dorwart MR, Shcheynikov N, Baker JM, Forman-Kay JD, Muallem S, Thomas PJ: Congenital chloride-losing diarrhea causing mutations in the stas domain result in misfolding and mistrafficking of slc26a3. J Biol Chem 2008;283:8711-8722.

6 Dirami T, Rode B, Jollivet M, Da Silva N, Escalier D, Gaitch N, Norez C, Tuffery P, Wolf JP, Becq F, Ray PF, Dulioust E, Gacon G, Bienvenu T, Toure A: Missense mutations in slc26a8, encoding a sperm-specific activator of cftr, are associated with human asthenozoospermia. Am J Hum Genet 2013;92:760-766.

7 Babu M, Greenblatt JF, Emili A, Strynadka NC, Reithmeier RA, Moraes TF: Structure of a slc26 anion transporter stas domain in complex with acyl carrier protein: Implications for e. Coli ychm in fatty acid metabolism. Structure 2010;18:1450-1462.

-8 Sharma AK, Ye L, Zolotarev AS, Alper SL, Rigby AC: Nmr assignment and secondary structure of the stas domain of rv1739c, a putative sulfate transporter of mycobacterium tuberculosis. Biomol NMR Assign 2009;3:99-102.

9 Sharma AK, Ye L, Baer CE, Shanmugasundaram K, Alber T, Alper SL, Rigby AC: Solution structure of the guanine nucleotide-binding stas domain of slc26-related sulp protein rv1739c from mycobacterium tuberculosis. J Biol Chem 2011;286:8534-8544.

$\checkmark 10$ Sharma AK, Ye L, Alper SL, Rigby AC: Guanine nucleotides differentially modulate backbone dynamics of the stas domain of the sulp/slc26 transport protein rv1739c of mycobacterium tuberculosis. Febs J 2012;279:420-436.

11 Pasqualetto E, Aiello R, Gesiot L, Bonetto G, Bellanda M, Battistutta R: Structure of the cytosolic portion of the motor protein prestin and functional role of the stas domain in slc26/sulp anion transporters. J Mol Biol 2010;400:448-462.

12 Seavers PR, Lewis RJ, Brannigan JA, Verschueren KH, Murshudov GN, Wilkinson AJ: Structure of the bacillus cell fate determinant spoiiaa in phosphorylated and unphosphorylated forms. Structure 2001;9:605-614.

13 Kovacs H, Comfort D, Lord M, Campbell ID, Yudkin MD: Solution structure of spoiiaa, a phosphorylatable component of the system that regulates transcription factor sigmaf of bacillus subtilis. Proc Natl Acad Sci USA 1998;95:5067-5071.

14 Masuda S, Murakami KS, Wang S, Anders Olson C, Donigian J, Leon F, Darst SA, Campbell EA: Crystal structures of the adp and atp bound forms of the bacillus anti-sigma factor spoiiab in complex with the anti-anti-sigma spoiiaa. J Mol Biol 2004;340:941-956.

15 Etezady-Esfarjani T, Placzek WJ, Herrmann T, Wuthrich K: Solution structures of the putative anti-sigmafactor antagonist tm1442 from thermotoga maritima in the free and phosphorylated states. Magn Reson Chem 2006;44:S61-70. 


\section{Cellular Physiology \\ and Biochemistry}

Cell Physiol Biochem 2014;33:605-620

\begin{tabular}{l|l} 
DOI: $10.1159 / 000358638$ & (C) 2014 S. Karger AG, Basel
\end{tabular}

16 Lindahl ER: Molecular dynamics simulations. Methods Mol Biol 2008;443:3-23.

17 Wishart DS, Sykes BD: The 13c chemical-shift index: A simple method for the identification of protein secondary structure using 13c chemical-shift data. J Biomol NMR 1994;4:171-180.

18 Sali A, Potterton L, Yuan F, van Vlijmen H, Karplus M: Evaluation of comparative protein modeling by modeller. Proteins 1995;23:318-326.

19 Van Der Spoel D, Lindahl E, Hess B, Groenhof G, Mark AE, Berendsen HJ: Gromacs: Fast, flexible, and free. J Comput Chem 2005;26:1701-1718.

20 Sharma AK, Rigby AC, Alper SL: Stas domain structure and function. Cell Physiol Biochem 2011;28:407422.

21 Laskowski RA, MacArthur MW, Moss DS, Thornton JM: Procheck: A program to check the stereochemical quality of protein structures. J Appl Cryst 1993;26:283-291.

22 Wiederstein M, Sippl MJ: Prosa-web: Interactive web service for the recognition of errors in threedimensional structures of proteins. Nucleic Acids Res 2007;35:W407-410.

23 Bowie JU, Luthy R, Eisenberg D: A method to identify protein sequences that fold into a known threedimensional structure. Science 1991;253:164-170.

-24 Emsley P, Lohkamp B, Scott WG, Cowtan K: Features and development of coot. Acta Crystallogr D Biol Crystallogr 2010;66:486-501.

25 Eyal E, Gerzon S, Potapov V, Edelman M, Sobolev V: The limit of accuracy of protein modeling: Influence of crystal packing on protein structure. J Mol Biol 2005;351:431-442.

-26 Oostenbrink C, Villa A, Mark AE, van Gunsteren WF: A biomolecular force field based on the free enthalpy of hydration and solvation: The gromos force-field parameter sets 53a5 and 53a6. J Comput Chem 2004;25:1656-1676.

27 Lemkul JA, Allen WJ, Bevan DR: Practical considerations for building gromos-compatible small-molecule topologies. J Chem Inf Model 2010;50:2221-2235.

-28 Anandakrishnan R, Aguilar B, Onufriev AV: H++ 3.0: Automating pk prediction and the preparation of biomolecular structures for atomistic molecular modeling and simulations. Nucleic Acids Res 2012;40:W537-541.

29 Molecular Operating Environment (MOE): Chemical Computing Group Inc, 2013;1010 Sherbooke St. West, Suite \#910, Montreal, QC, Canada, H3A 2R7, 2013.

-30 Krivov GG, Shapovalov MV, Dunbrack RL Jr: Improved prediction of protein side-chain conformations with scwrl4. Proteins 2009;77:778-795.

31 Hess B, Bekker H, Berendsen HJC, Fraaije JGEM: LINCS: A linear constraint solver for molecular simulations. J Comput Chem 1997;18:1463-1472.

-32 Essmann U, Perera L, Berkowitz ML, Darden T, Lee H, Pedersen LG: A smooth particle mesh Ewald method. J Chem Phys 1995;103:8577-8593.

-33 Koradi R, Billeter M, Wuthrich K: Molmol: A program for display and analysis of macromolecular structures. J Mol Graph 1996;14:51-55, 29-32.

-34 Humphrey W, Dalke A, Schulten K: Vmd: Visual molecular dynamics. J Mol Graph 1996;14:33-38, 27-38.

-35 Bai JP, Navaratnam D, Samaranayake H, Santos-Sacchi J: En block c-terminal charge cluster reversals in prestin (slc26a5): Effects on voltage-dependent electromechanical activity. Neurosci Lett 2006;404:270275.

-36 Shcheynikov N, Yang D, Wang Y, Zeng W, Karniski LP, So I, Wall SM, Muallem S: The slc26a4 transporter functions as an electroneutral cl-/i-/hco3- exchanger: Role of slc26a4 and slc26a6 in i- and hco3- secretion and in regulation of cftr in the parotid duct. J Physiol 2008;586:3813-3824.

-37 Reimold FR, Heneghan JF, Stewart AK, Zelikovic I, Vandorpe DH, Shmukler BE, Alper SL: Pendrin function and regulation in xenopus oocytes. Cell Physiol Biochem 2011;28:435-450.

-38 Nygaard R, Zou Y, Dror RO, Mildorf TJ, Arlow DH, Manglik A, Pan AC, Liu CW, Fung JJ, Bokoch MP, Thian FS, Kobilka TS, Shaw DE, Mueller L, Prosser RS, Kobilka BK: The dynamic process of beta(2)-adrenergic receptor activation. Cell 2013;152:532-542. 